\title{
Surface Modification Using Nano-phase Structures of Epoxy/block Copolymer Blends for Electroless Copper Plating
}

\author{
Tomohiro Mori ${ }^{1}$, Masanobu Endo ${ }^{1}$ and Hajime Kishi ${ }^{1 *}$
}

Key words : Epoxy, Block copolymer, Phase structure, Electroless plating, Peel strength

\section{Introduction}

Adhesion between electroless copper plating and polymer substrates of printed circuit boards has been an important issue. At present, the good adhesion can be achieved using the pre-formed rough surfaces in $\mu \mathrm{m}$ scale on the substrates ${ }^{11}$. However, considering for more-miniaturization trend with the high performance of semiconductor integrated circuit, the advanced polymer surfaces having the less-roughness were required with maintaining the good adhesion of the copper plate layer.

Several nano-phase structures of the cured epoxy/ PMMA- $b$-PnBA- $b$-PMMA tri-block copolymer (BCP) blends were reported by one of the authors ${ }^{2,3)}$. We have considered that the sub-micron roughness might be constructed by the selective etching of the elastomer phase in the epoxy/BCP nano-alloys. In this study, the cured epoxy/BCP blends were examined as the polymer substrates for the electroless copper plating.

\section{Experimental}

\subsection{Materials}

Diglycidyl ethers of bisphenol-A (DGEBA, epoxy equivalent weight: $189 \mathrm{~g} / \mathrm{eq}$ ) was utilized as the epoxy resin. Stoichiometric amount of phenol novolac (PN, hydroxyl group equivalent weight: $105 \mathrm{~g} /$ eq.) was used as the curing agent.

1 Graduate School of Engineering, University of Hyogo, 2167, Shosha, Himeji, Hyogo, 671-2201 Japan,

Tel: +81-79-267-4843, Fax: +81-79-267-4843,

E-mail: kishi@eng.u-hyogo.ac.jp

(Received : January 6, 2015)
Tri-phenyl phosphine (TPP) worked as a catalyst used with the PN. The amount of the TPP was 0.5 parts per hundred resin.

The PMMA- $b$-PnBA- $b$-PMMA tri-block copolymer (BCP: weight-average molecular mass $\mathrm{Mw}=72000$, PnBA content in the BCP was 77 wt $\%, \mathrm{Mw} / \mathrm{Mn}=1.19$ ) were dissolved in the DGEBA. The blends were precured using $\mathrm{PN} / \mathrm{TPP}$ at $120^{\circ} \mathrm{C}$ for $2 \mathrm{~h}$, and post cured at $150^{\circ} \mathrm{C}$ for $2 \mathrm{~h}$. The content of the $\mathrm{BCP}$ was $10 \mathrm{wt} \%$ in the cured blends.

\subsection{Surface treatment for substrates and the electroless copper plating}

The cured epoxy/BCP blends were etched using oxygen plasma generator SAMCO PT-500S. Oxidation treatment was conducted using UV-ozone surface processor (UV wavelength: $253.7 \mathrm{~nm}$, Sen Lights Corporation, UVE-200G-SSII). The cured epoxy substrates were subjected to electroless plating using the copper plating agent (OPC-750, Process-M) from Okuno Chemical.

\subsection{Evaluation methods}

Scanning electron microscope (SEM), atomic force microscope (AFM), and Fourier transform infrared spectroscopy (FT-IR, ATR method) were applied to examine the surface of the epoxy substrates. Peel strengths of the copper plating layer on the polymer substrates was measured using $90^{\circ}$ peel configuration by a tensile tester (Shimadzu, sm-500n-168).

\section{Results and Discussion}

Fig. 1 shows a SEM image (reflection electron mode) of a surface of epoxy/BCP blend. The relatively bright 
phases consist of the PnBA stained by $\mathrm{RuO}_{4}$, and the dark matrix mainly consists of the epoxy thermoset. The curved lamella type nano-phase structure were observed ${ }^{2,3)}$. The nano-phase structure was formed by the self-assembly mechanism of the epoxy/BCP blend ${ }^{2)}$. The PnBA elastomer phase was expected to be etched selectively by the oxygen-plasma treatment. In fact, the curved lamellar-type concave structure was observed after the plasma etching (Fig. 2). Fig. 3 shows the effect of the plasma etching to make the surface roughness of the epoxy/BCP blends. The roughness increased with increasing the plasma treatment, although the etching was not observed on the pure epoxy substrates.

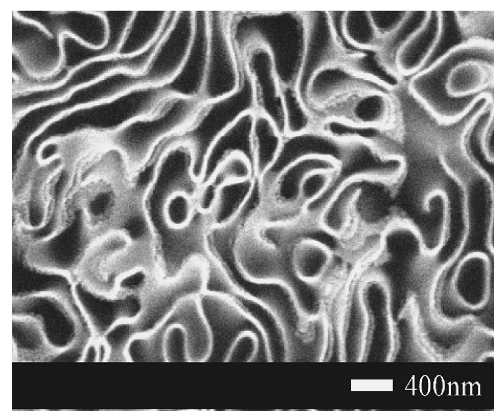

Fig. 1 "Curved lamellar" nano-phase structure of epoxy/ BCP (10wt\% BCP in the cured epoxy blend)

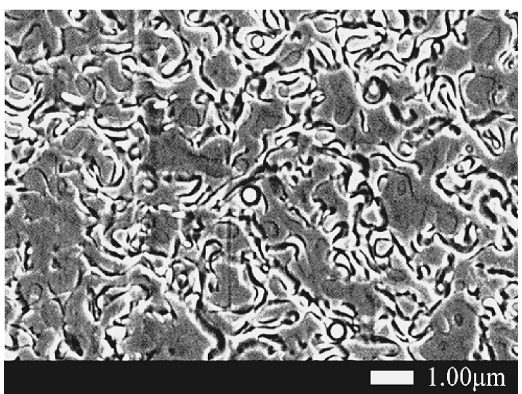

Fig. 2 Plasma-etched surface of the epoxy/BCP blend

Fig. 4 shows the effect of the surface roughness (Rzjis) for the peel strength of the copper plating layer on the epoxy/BCP substrates. The peel strengths of the electroless copper plating increased by the "anchor effect" of the plasma-etched epoxy/BCP substrates. However, the simple concave formation was not enough to achieve the required peel strength (more than $7 \mathrm{~N} / \mathrm{cm}$ ).

Therefore, UV-ozone oxidation was applied as the additional treatment, because the increased polarity was

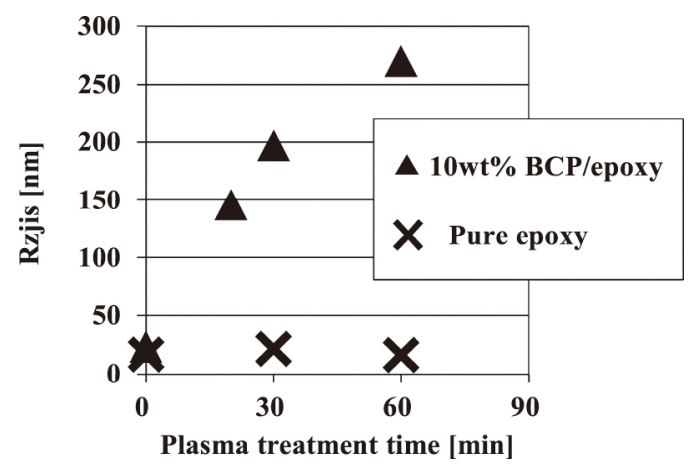

Fig. 3 Effect of the plasma treatment on the surface roughness of the epoxy/BCP blends and pure epoxy substrates

expected to work for the wettability of the plating agent on the plasma-etched epoxy substrates. The FTIR spectra indicated the oxidation, as evidenced by the increased absorbance ratio on $\mathrm{C}=\mathrm{O}$ /benzene ring from 0.3 to 0.6 . As the results, the peel strength has reached $8.1 \mathrm{~N} / \mathrm{cm}$ in the best mode, with keeping the surface roughness (Rzjis) of $93 \mathrm{~nm}$, as shown in Fig. 4 . It should be noted that the peel strength of the nonetched surface was only $0.3 \mathrm{~N} / \mathrm{cm}$, even after the same UV-ozone oxidation. Big synergistic effect of "nanoetching" and "oxidation" enabled the high peel strength of the copper plating. The plausible mechanism would be the increased wettability of the waterbased plating agents into the nano-concaved structures on the treated substrates.

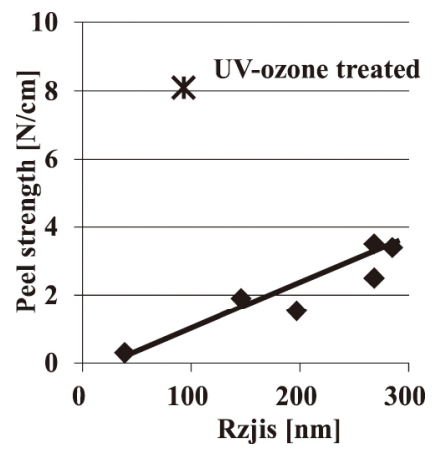

Fig. 4 Effect of the surface roughness \& ozone oxidation for the peel strength of the elecroless copper plating on the epoxy/BCP substrates

\section{References}

1) E. Njuhovic et al., Surface \& Coatings Technology, 232 , 319 (2013).

2) H. Kishi et al., Polymer, 52, 760 (2011).

3) H. Kishi et al., Express Polymer Letters, 9 (1), 23 (2015). 